\title{
Design and implementation of wellsite data transmission system based on C/S
}

\author{
Baolei Ma1, a Yanmei Zhang1, b \\ ${ }^{1}$ China University of Geosciences (Beijing), 20, Chengfu Road, Haidian District, Beijing, 100083, \\ China \\ a837907970@qq.com, bymzhang@cugb.edu.cn
}

Keywords: WITS; C/S; Socket programming; Database; Real-time

\begin{abstract}
The paper introduces the data transmission system in the Petroleum Industry with the help of programming systematically. The wellsite data could be available for the usage of other Petroleum Service Departments by using this system. The perfection of Internet technology enables network programming to become a major way of sharing information. There are so many kinds of programming languages with the continuous development of IT industry, and the essence of those languages is largely identical but with minor differences. The purpose of this project is to generalize a kind of software model that combining with the characteristics of the wellsite data. It will be added some new features on the basis of traditional communication model based on $\mathrm{C} / \mathrm{S}$ structure. There are also many kinds of data transmission software, but the core technology is socket programming [1]. Now, we will induce a new model that having specific structure. The design of wellsite data transmission system is based on this new model. The paper will go through four parts as follows: Analysis, Design, Implementation and Testing.
\end{abstract}

\section{Introduction}

As economic growth, especially the development of the computer industry, the relationship between computer applications and various industries is getting closer. Petroleum Industry in China is in the initial stage, so the subject of wellsite data transmission system is of practical significance. It does help for the communication between various Petroleum Service Departments.

Designing a software project, the data sending terminal handles data-editing and data-decoding. What is more, the data receiving terminal handles receiving socket communication packets and storing data into database. The programming based on $\mathrm{C} / \mathrm{S}$ structure is easy to achieve this kind of transfer mechanism. The server program is responsible for sending wellsite data and the client program is to complete the data receiving.

Geosteering technology, relying on a huge amount of real-time wellsite data, is playing an increasingly essential role in oil exploration. International Petroleum companies focus on this research field, also it brings ecological and economic benefits. WITS (Wellsite Information transfer Standard) by the International Petroleum Association [2], it offers a model for programming. The overall software model integrated the features of WITS on the basis of the traditional model of data transmission in the paper.

\section{Analysis}

Software implementation starts with system analysis. It is well known that a good software program is dependent on robust software analysis. In the analysis process, the programmers could make the correct feasibility estimate and determine the implementation approach by project requirements. Now the paper will give analyses from three aspects.

Consumer perspective. Considering the needs of consumers, they would not let programmers to develop specific software program for each instruments. Meanwhile, easy-manipulating is always the golden rule for users. All they want is to complete data transmission from different instruments and data storage with simple steps. 
Developer perspective. Analyzing from the perspective of developers, Microsoft(R) Windows $\mathrm{XP}(\mathrm{TM})$ is development and runtime environment of the application program, in order to get a better compatibility, software development tools is visual studio 2008(C\#)[3], including interfacial design, socket programming and multithreads programming, etc. As for database, we decided to use Sql Server 05 for software development. Comparing with programming in $\mathrm{B} / \mathrm{S}, \mathrm{C} / \mathrm{S}$ structure performs very well with little responsive time [4], which qualifies with the strict need of real-time wellsite data.

Technological auxiliary means. The realization of the main function needs to use a variety of techniques. As the Internet technology getting mature gradually, Internet has become the carrier of information exchange. Further more information transfer on the Internet by using of such as network communication protocol (TCP/IP), socket communication technology, as well as $\mathrm{C} / \mathrm{S}$ structure application, etc. Internet did not have the ability to become the carrier until all these technologies were integrated together [5].

Firstly, C/S structure application is generally applied to the special network a small scale network environment. This kind of application program could provide connection and data exchange services between local area network (LAN) by the way of via a dedicated server. Secondly, the growing network consumption reduces the overhead cost of online services. As a result, many businesses are also more and more depend on online services. For example, the popular online shopping reflects the efficient and convenient features of electronic commerce. Finally, we know that the socket was not necessarily associated with TCP/IP. If a program creates a socket object to listen on port 80 , and this declares TCP/IP protocol stack for the possession of the port. In other words, it is socket interface that makes the software connected to the Internet.

\section{Design}

Wellsite data is delivered from one computer to another in the case of Internet access. Client, as the data receiving terminal, follows the requirements of users to store data into relevant databases; Server, as the data sending terminal, implements data-editing by users on condition that the wellsite data itself is not enough robust and data-encoding, etc. Figure. 1 shows the communication model based on $\mathrm{C} / \mathrm{S}$ of this system, depending on socket programming and IP addressing.

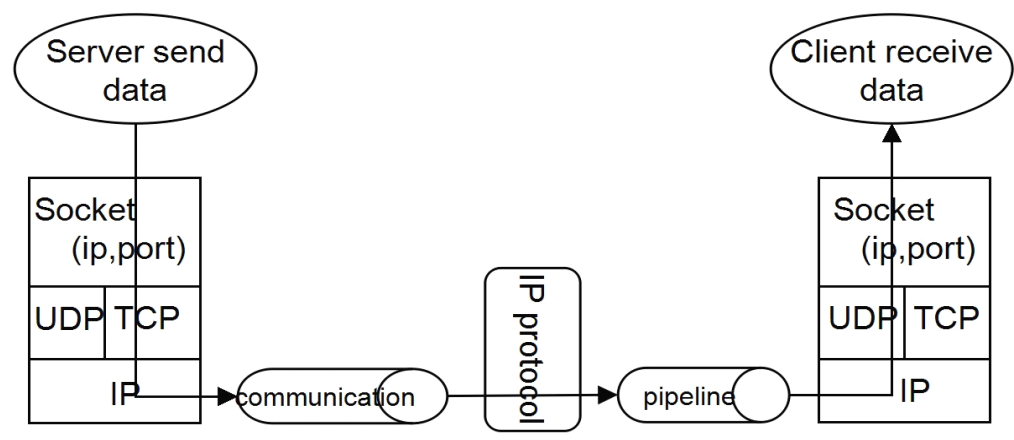

Fig. 1, socket communication model

Design of client application. Client application takes large amount of tasks including configuring database and setting communication sites (IP address and port). A large part of information to complete data transmission should be set up in client application. It is no doubt that software mush keep to simply interact with users. In that case, users just need to modify these parameters to complete data transmission though the environment on which the wellsite facilities running needs to change. In addition, network connection information and database storing information should be configured directly by users too.

Design of server application. Server application sends wellsite data to client terminal. Wellsite facilities will generate real-time geological data when drilling rig is working and then retrieving geological data by key-point (time or depth) in a disperse way. After that, add marks upon those data following WITS specification. Of course, users can also modify the data before that. If developers do not consider data-editing, a lot of design work will be reduced. 
Design of functional module. The functional module designing has a particularly important role throughout the design phase of the project [6]. Software function of wellsite data transmission system mainly includes data store, wellsite data manipulation and socket interface designing. The functional framework of this project is composed of WITS Data Processing Module, Socket Communication Module and Database Storage Module as shown in the figure.2.

1) WITS Data Processing Module: It is should be emphasized that this module can be called by either client or server application. The difference is the server requires data-editing function while the client requires data-decoding function out of the module. By modifying this module, the software could also adapt to other places, other information. That is to say the software becomes easy to maintain.

2) Socket Communication Module: This module is the most essential part of the same is also the largest workload. It deals with building network connection and reading/writing files, etc. When using this module, the program will create a socket object. It has mentioned above that the socket object is interface to connect application program and network. So the purpose of this module is to link up the sending end and the receiving end for communication. In addition, there are two other sub modules, user management module and read/write file module.

3) Database Storage Module: This module processes all database connection problems. It is just like some kind of interface to build connection between software and database.

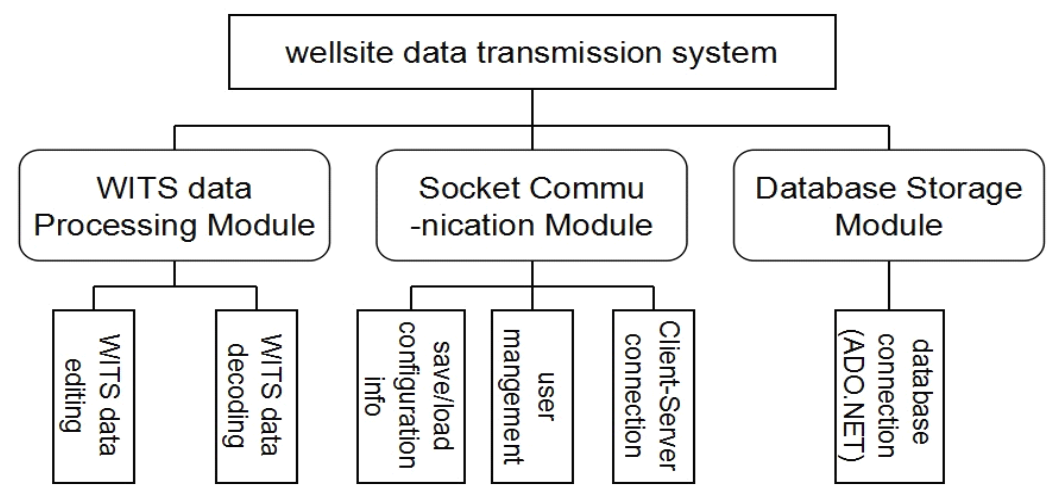

Fig. 2, the architecture diagram of system functions

Design of database. The database at the receiving end is used for storing wellsite data. To design database tables, the programmers need to comply with WITS.

\section{Implementation}

To the overall framework of this project, software implementation is based on $\mathrm{C} / \mathrm{S}$ structure of the socket network programming. There are some descriptions of the three functional modules.

Client implements two kinds of receiving mode. One mode is client sends connection request first and then receive data when communication path established; another mode is client opens monitoring function, it will send a feedback message once receiving a connection request and then the path was built. There needs to clearly point out that the key to distinguish between client and server is which one is monitoring. But, we called the data source as server.

Wellsite data is packed with a certain media format. This kind of data is called wits-data. In order to achieve the editing and translation of the data, acquiring collection classes, such as Hashtable, ArrayList. One benefit of those is that you can freely add and remove elements to the object without having to worry about memory management. In addition, it is very convenient to retrieve these collection objects. These features are used in the user management and establish a connection.

The process of filtering wellsite data complete getting rid of obvious wrong data and gets them corrected. Firstly, data from the server is stored in local variable DataTable in 2D form. DataTable object is bound to DataGridView interface control. When the data has been modified in a DataGridView object, the DataTable object gets cascading updated automatically by traversing every item in and modify it. But, real-time may be disappeared. 
We ought to use WITS, when designing the database in client terminal. WITS, it is regulated the structure of 25 pieces of sheets that cover every type of wellsite data. Obviously, the goal of such a database design is to implement simple matching between wits-data names and database field names. Database access model is ADO.NET [7,8], which is getting more and more popular by features as high performance, simple manipulation, less memory usage, etc.

Software configuration information will be saved as a system file. We do not want to see that connection is interrupted suddenly in the process of data transmission. So, the software will need to have the memory function to record the state of transmission once interrupt appears. These messages are not saved in database, but saved into configuration files by using file read and write.

\section{Testing}

The paper focuses on overall architecture of wellsite data transmission system. It mainly introduced analysis phase, design phase and implementation phase of the software implementation. However, multithreaded programming section is left out during the software development procedure for it requires high level programming skills and algorithm designs from developers to make the program optimization. The main challenge of this project goes to dealing with multithreaded deadlock occurring on main thread when users are interacting with software. So the client software will have to solve conflicts as database storage module sharing also known as resource usage upon multipoint connection between clients and servers or accepting data sent from different servers.

\section{Summary}

To sum up, socket programming based on $\mathrm{C} / \mathrm{S}$ structure is featured for easier development and lower cost. Wellsite data transmission system is able to meet the requirement of real-time drilling data sharing. What is more, we users only need to modify a configuration file to make it compatible for various environments. Whenever the system gets started with this configuration file loaded to satisfy such a feature in a general way. Actually, this kind of design is supposed to be applied to even broader field of production.

\section{References}

[1] Jun Liu, Gangfeng Yan. Network Programming Technique Based on Socket. Natural Science, Vol.3, No.3 (2004).

[2] Information on http://wenku.baidu.com/view/18e5cf87b9d528ea81c779b4.html.

[3] Lixia Liu, Junmin Li. C\# sample development. Tsinghua University Press, 2010, 34-113.

[4] Wenbo Huang, Analysis between C/S and B/S. Natural Science, Vol.25, No.4 (2006).

[5] Eddie Law K L, Leung R. A design and implementation of active network socket programming [J]. Microprocessors and Microsystems, 2003, 27(5): 277-284.

[6] Yingchun Deng, Song Han, Tianshun Xu. Object-Oriented and Classical Software Engineering. China Machine Press, 2007, 249-306.

[7] Baoxiang Wang. Study of Database Access Technology using ADO.NET method. Computer Application and Software, Vol.21, No.2 (2004).

[8] HUA G, LIU W. Performance Optimizing in Database Accessing Based on ADO.NET [J][J]. Application Research of Computers, 2004, 6: 076.

\section{Acknowledgments}

This work is supported by the National Key Basic Research Development Program (National Development Plan-973Plan) under Grant Nos.2011CB201103. 